\title{
Targeting the Id1-Kif11 Axis in Triple-Negative Breast Cancer Using Combination Therapy
}

\author{
Archana P. Thankamony ${ }^{1,2, t, \ddagger}$, Reshma Murali ${ }^{1,+, \ddagger}$, Nitheesh Karthikeyan ${ }^{1}$, \\ Binitha Anu Varghese ${ }^{1}$, Wee S. Teo ${ }^{3}$, Andrea McFarland ${ }^{3}$, Daniel L. Roden ${ }^{3,4}$, \\ Holly Holliday 3,4 ${ }^{(D}$, Christina Valbirk Konrad ${ }^{3}\left(\mathbb{D}\right.$, Aurelie Cazet ${ }^{3,4}$, Eoin Dodson ${ }^{3,4}$, \\ Jessica Yang ${ }^{3}$, Laura A. Baker ${ }^{3,4}$, Jason T. George ${ }^{5,6} \mathbb{D}^{\mathbb{D}}$, Herbert Levine ${ }^{5,7}(\mathbb{D}$, \\ Mohit Kumar Jolly ${ }^{8}$ (D), Alexander Swarbrick ${ }^{3,4}$ and Radhika Nair 1,*(D) \\ 1 Cancer Research Program, Rajiv Gandhi Centre for Biotechnology, Kerala 695014, India; \\ archanapt@rgcb.res.in (A.P.T.); reshmamurali@rgcb.res.in (R.M.); nitheeshpvk@gmail.com (N.K.); \\ binitha.a.v@gmail.com (B.A.V.) \\ 2 Manipal Academy of Higher Education (MAHE), Manipal, Karnataka 576104, India \\ 3 The Kinghorn Cancer Centre and Cancer Research Theme, Garvan Institute of Medical Research, \\ Darlinghurst, NSW 2010, Australia; weeteo2011@gmail.com (W.S.T.); a.mcfarland@garvan.org.au (A.M.); \\ d.roden@garvan.org.au (D.L.R.); h.holliday@garvan.org.au (H.H.); c-v-k@hotmail.com (C.V.K.); \\ a.cazet@garvan.org.au (A.C.); e.dodson@garvan.org.au (E.D.); j.yang@garvan.org.au (J.Y.); \\ laura_baker11@hotmail.com (L.A.B.); a.swarbrick@garvan.org.au (A.S.) \\ 4 St Vincent's Clinical School, Faculty of Medicine, University of New South Wales, \\ Darlinghurst, NSW 2052, Australia \\ 5 Center for Theoretical Biological Physics, Rice University, Houston, TX 77005, USA; jtg6@rice.edu (J.T.G.); \\ herbert.levine@rice.edu (H.L.) \\ 6 Medical Science Training Program, Baylor College of Medicine, Houston, TX 77005, USA \\ 7 Departments of Bioengineering and Physics, Northeastern University, Boston, MA 02115, USA \\ 8 Centre for BioSystems Science and Engineering, Indian Institute of Science, Bangalore 560012, India; \\ mkjolly@iisc.ac.in \\ * Correspondence: radhikanair@rgcb.res.in; Tel.: +91-471-2781251 \\ + Equal first authors. \\ $\ddagger$ Present address: Rajiv Gandhi Centre for Biotechnology, Kerala 695014, India.
}

Received: 25 June 2020; Accepted: 27 August 2020; Published: 8 September 2020

\begin{abstract}
The basic helix-loop-helix (bHLH) transcription factors inhibitor of differentiation 1 (Id1) and inhibitor of differentiation 3 (Id3) (referred to as $I d$ ) have an important role in maintaining the cancer stem cell (CSC) phenotype in the triple-negative breast cancer (TNBC) subtype. In this study, we aimed to understand the molecular mechanism underlying Id control of CSC phenotype and exploit it for therapeutic purposes. We used two different TNBC tumor models marked by either Id depletion or $I d 1$ expression in order to identify $I d$ targets using a combinatorial analysis of RNA sequencing and microarray data. Phenotypically, Id protein depletion leads to cell cycle arrest in the G0/G1 phase, which we demonstrate is reversible. In order to understand the molecular underpinning of Id proteins on the cell cycle phenotype, we carried out a large-scale small interfering RNA (siRNA) screen of 61 putative targets identified by using genomic analysis of two Id TNBC tumor models. Kinesin Family Member 11 (Kif11) and Aurora Kinase A (Aurka), which are critical cell cycle regulators, were further validated as Id targets. Interestingly, unlike in Id depletion conditions, Kif11 and Aurka knockdown leads to a G2/M arrest, suggesting a novel Id cell cycle mechanism, which we will explore in further studies. Therapeutic targeting of Kif11 to block the Id1-Kif11 axis was carried out using small molecular inhibitor ispinesib. We finally leveraged our findings to target the Id/Kif11 pathway using the small molecule inhibitor ispinesib in the Id+ CSC results combined with chemotherapy for better response in TNBC subtypes. This work opens up exciting new possibilities of targeting Id targets such as Kif11 in the TNBC subtype, which is currently refractory to chemotherapy. Targeting
\end{abstract}


the Id1-Kif11 molecular pathway in the Id1+ CSCs in combination with chemotherapy and small molecular inhibitor results in more effective debulking of TNBC.

Keywords: cancer stem cells; chemoresistance; self-renewal; combination therapy; Id1; Kif11

\section{Introduction}

Breast cancer is a heterogeneous disease with different molecular subtypes displaying distinct outcomes $[1,2]$. The triple-negative breast cancer (TNBC) subtype does not express molecular markers such as estrogen receptor (ER) and human epidermal growth factor receptor 2 (HER2) that are the basis of targeted therapies in other molecular subtypes of breast cancer [3,4]. Consequently patients presenting with TNBC are left with few therapeutic choices, resulting in lower five-year survival rates when compared to the other subtypes [3]. Hence, there is an urgent need to understand the molecular basis of TNBC in order to identify new drug targets.

The critical role of a subpopulation of cells termed cancer stem cells (CSCs) in self-renewal, chemoresistance, and metastasis has assumed clinical importance in breast cancer $[5,6]$. The inhibitor of differentiation (Id) proteins are negative regulators of the basic helix-loop-helix (bHLH) transcription factors [7]. The Id proteins are important for maintaining the CSC population and therefore tumor progression in TNBC. We have previously shown that $I d 1 / 3$ (collectively known as $I d$ ) are critical for the CSC-associated phenotypes in the TNBC molecular subtype [1]. Genetic screen analysis of Id knockdown (Id KD) and Id1 expression models led to the identification of Kif11 and Aurka as putative Id targets in this study.

The detailed mechanism by which Id controls the cell cycle is not clear, although Id is known to impact the pathway via decreased expression of cyclins $D 1$ and $E$, reduced phosphorylation of $R b$ as well as reduced cyclin E-Cdk2 activity [8]. In this work, we show how Id acts as a central focal point to coordinate the cell cycle genes Kif11 and Aurka and demonstrate that Id KD leads to cell cycle arrest in the G0/G1 phase of the cell cycle. Interestingly, we found that the depletion of Kif11 and Aurka independently did not phenocopy the G0/G1 arrest we observed in Id KD cells. We demonstrated that Id KD puts the brakes on the cell cycle, resulting in a state of arrest at the G0/G1 phase via impacting cell cycle molecules. Moreover, we demonstrated that $I d$ is a critical driver of self-renewal, acting via Kif11 and Aurka. We found that the Id-expressing tumor cells were resistant to chemotherapy, which forms the first line of treatment in TNBC. Interestingly, treatment with ispinesib, a small molecule inhibitor against Kif11, resulted in the reduced expression of Id in these cells. We finally exploited this finding to treat tumor cells with the chemotherapeutic drug paclitaxel, combined with ispinesib to ablate the Id-expressing chemoresistant tumor cells along with bulk tumor cells, leading to more effective therapeutic targeting in the TNBC subtype.

\section{Methods}

\subsection{Cell Culture}

$4 \mathrm{~T} 1$ cells were sourced from American Type Cell Culture Collection (ATCC) and cultured as per specifications. The 4T1 Id1GFP cells were generated by lentiviral infection of the 4T1 cells with the Id1 GFP reporter, as reported previously [1].

\subsection{Immunofluorescence}

Cells were fixed in 4\% paraformaldehyde (TCL119-100ML, Himedia, Mumbai, India), permeabilized with $0.2 \%$ TritonX-100 (T9284, Sigma-Aldrich, St. Louis, Missouri, USA), and blocked with $1 \%$ bovine serum albumin (BSA) (A7906, Sigma-Aldrich) in phosphate buffered saline (PBS) (Gibco, Grand Island, NY, USA)for $1 \mathrm{~h}$ at room temperature. The cells were then incubated with 
primary antibody at $4{ }^{\circ} \mathrm{C}$ overnight, followed by secondary antibody for $1 \mathrm{~h}$ at room temperature. The nuclei were stained with 4',6-diamidino-2-fenylindool (DAPI; Sigma-Aldrich). Images were taken by confocal microscopy (Leica Microsystems, Wetzlar, Germany).

\subsection{Cell Cycle Analysis}

Cells were harvested and incubated with Hoechst 33342 (H3570, Invitrogen, CA, USA; $4 \mathrm{ug} / \mathrm{mL}$ ) at $37^{\circ} \mathrm{C}$ for $30 \mathrm{~min}$. The cell cycle distribution was determined with a flow cytometer (BD FACS Aria III, BD Biosciences, San Jose, CA, USA). The data were analyzed using the BD FACS analyzer software.

\subsection{Quantitative Real-Time PCR ( $q R T-P C R)$}

Total RNA was isolated using TRIzol Reagent (15596026, Invitrogen, Carlsbad, CA, USA)), and complementary DNA (cDNA) was synthesized using High-Capacity cDNA Reverse Transcription Kits (4368814, Applied Biosystems, Foster City, CA, USA). Real-time PCR was performed on the QuantStudio 7 Flex Real-Time PCR System (Applied Biosystems) using Power SYBR ${ }^{\circledR}$ Green PCR Master Mix (Applied Biosystems). All target gene expression levels were normalized to $\beta$-actin. The relative fold change was determined by $2^{-\Delta \Delta C T}$ method, as described previously [9]. The sequences of primers used to detect target mouse genes in qRT-PCR are listed in Table S4.

\subsection{Flow Cytometry}

Adherent cells were trypsinized and washed with PBS and blocked with Fc Receptor (FcR) blocking reagent (130-092-575, Miltenyi Biotec, Bergisch Gladbach, Germany) for $1 \mathrm{~h}$. The cells were then incubated with fluorescein isothiocyanate (FITC)-conjugated anti-mouse cluster of differentiation 29 (CD29) (130-102-975, Miltenyi Biotec) and R-phycoerythrin (PE)-conjugated anti-human cluster of differentiation 24 (CD24) (130-103-371, Miltenyi Biotec) antibodies (1:50 dilution) for 20 min at $4{ }^{\circ} \mathrm{C}$ in the dark. Cells were washed twice with PBS and resuspended in $500 \mu \mathrm{L}$ of fluorescence activated cell sorting (FACS) buffer prior to analysis on a FACS flow cytometer (BD FACS Aria III, BD Biosciences).

\subsection{Microarray and Bioinformatics Analysis}

Total RNA from the samples were isolated using a Qiagen RNeasy min ikit (Qiagen, Doncaster, VIC, Australia). cDNA synthesis, probe labelling, hybridization, scanning, and data processing were all conducted by the Ramaciotti Centre for Gene Function Analysis (The University of New South Wales). Gene expression profiling was performed using the AffymetrixGeneChip Gene 1.0 ST Array, a whole-transcript array that covers 28,000 coding transcripts and 7000 non-coding long intergenic non-coding transcripts. Data analysis was performed using the Genepattern software package from the Broad Institute. Three different modules, Hierarchical Clustering Viewer, Comparative Marker Selection Viewer, and Heatmap Viewer were used to visualize the data. In addition to identifying candidate molecules and pathways of interest, Gene Set Enrichment Analysis (GSEA) (http://www.broadinstitute.org/gsea) was performed using the GSEA pre-ranked module. Briefly, GSEA compares differentially regulated genes in an expression profiling dataset with curated and experimentally determined sets of genes in the MSigDB database to determine if certain sets of genes are statistically over-represented in the expression profiling data.

\section{7. siRNA Screen to Assess Proliferation}

Reverse transfection of $4 \mathrm{~T} 1$ cells in 384-well plates was performed with 400 cells and $0.08 \mu \mathrm{L}$ Dharmafect1 per well using a Caliper Zephyr and Biotek EL406 liquid handling robots. Media was changed at $24 \mathrm{~h}$ post-transfection. CellTiter-Glo ${ }^{\circledR}$ assay (Promega, Madison, WI, USA) was performed using a BMG Clariostar plate reader (luminescence assay). 4T1 cells were reverse-transfected with a 40nM siGENOMESMART pool siRNA against each of the 57 candidate genes. Cell viability was quantified at $72 \mathrm{~h}$ post-transfection using the CellTiter-Glo ${ }^{\circledR}$ assay (Table S3). IncuCyte ZOOM live cell 
imaging every $2 \mathrm{~h}$ was also performed, which allowed us to quantify cell growth (confluence) over time throughout the experiment.

Final data presented were generated from three biological replicates, each consisting of two technical replicates. Viability measurements were normalized to the treatment-matched scrambled control after subtracting the blank empty wells.

\subsection{Western Blotting}

Cells were lysed with radio immunoprecipitation assay (RIPA) buffer (R0278-50ML, Sigma-Aldrich) containing 1X complete protease inhibitor cocktail (P8340-5ML, Sigma-Aldrich). Protein concentrations were determined using Pierce ${ }^{\mathrm{TM}}$ BCA Protein Assay Kit kit (23225, Thermofisher scientific, Waltham, Massachusetts, USA). Proteins were separated by sodium dodecyl sulfate polyacrylamide gel electrophoresis (SDS-PAGE) and transferred onto $45 \mu \mathrm{m}$ polyvinylidene difluoride (PVDF) membrane (1620177, Biorad, Hercules, California, USA). Membranes were blocked using 5\% non-fat dry milk in Tris-buffered saline with $0.1 \%$ Tween 20 (P1379, Sigma) and probed with the respective primary and secondary antibodies. The signal was detected based on enhanced chemiluminescence (ECL) using Clarity Western ECL Substrate (1705061, Bio-Rad, Berkeley, CA, USA. $\beta$-actin was used as a protein loading control. Antibodies used in this study are listed in Table S5.

\subsection{MTT Assay}

$4 \mathrm{~T} 1$ cells were seeded at a density of 500 cells per well in a 96-well plate. When the cells became $80 \%$ confluent, we added freshly prepared 3-(4,5-Dimethylthiazol-2-yl)-2,5-Diphenyltetrazolium Bromide (MTT) reagent (M6494, Thermofisher Scientific, Hyderabad, India) $(5 \mathrm{ug} / \mathrm{mL})$ to the culture media. The plates were incubated for $2 \mathrm{~h}$ in the dark at $37^{\circ} \mathrm{C}$. The media-containing reagent was removed from each well and $100 \mu \mathrm{L}$ of DMSO was added to each well. Absorbance reading was taken using a TECAN microplate reader at $570 \mathrm{~nm}$.

\subsection{IC 50 Values for Chemotherapeutic Drugs}

$4 \mathrm{~T} 1$ cells were harvested and seeded 1000 cells per well in a 96-well plate. When the cells became $20 \%$ confluent, culture media was removed and replenished with media containing the chemotherapeutic drugs paclitaxel $(10 \mathrm{nM})$ and doxorubicin $(30 \mathrm{nM})$; small molecular inhibitors ispinesib $(5 \mathrm{nM})$, alisertib $(100 \mathrm{nM})$, and MTT reading was taken $48 \mathrm{~h}$ post-drug treatment.

\subsection{Tumorsphere Assay}

4T1 cells were put into the tumorsphere assay, as described previously [1]. Paclitaxel and ispinesib were added at a concentration of $10 \mathrm{nM}$ and $5 \mathrm{nM}$, respectively, to assay for the effect of these drugs on self-renewal.

\subsection{The Kaplan-Meier Plotter}

The association of ID1 (OMIM accession number: 600349) and KIF11 (OMIM accession number: 148760) gene expression with the relapse-free survival (RFS) of breast cancer patients was analyzed using the Kaplan-Meier Plotter (KM Plotter, Semmelweis University, Budapest, Hungary) database (http://kmplot.com/analysis/) [10]. The gene expression and survival data are derived from Gene Expression Omnibus (GEO). KM Plotter is a manually curated database handled by the PostgreSQL server that integrates gene expression with clinical data. The patient cohorts were split on the basis of median gene expression by auto-select best cut-off. The ID1 and KIF11 genes were entered into the KM Plotter database and the RFS was determined for the TNBC subtype $(n=198$ patients). To perform multiple gene analysis, the mean expression of ID1 and KIF11 were used. The hazard ratio (HR) with $95 \%$ confidence and $p$-values were obtained from the KM Plotter. $p$-values $<0.05$ were considered as statistically significant. 


\subsection{Statistical Analysis}

Statistical analyses were performed using GraphPad Prism 6 (GraphPad Software, Inc., San Diego, CA, USA). All in vitro experiments were performed in three biological replicates each with two or more technical replicates. Data represented are means \pm standard deviation. Statistical tests used are unpaired Student's $t$-test and two-way ANOVA. $p$-values $<0.05$ were considered statistically significant with ${ }^{*} p<0.05,{ }^{* *} p<0.01,{ }^{* * *} p<0.001,{ }^{* * * *} p<0.0001$.

\section{Results}

\subsection{Id Depletion Leads to a G0/G1 Cell Cycle Arrest that Is Reversible}

It has been previously demonstrated that $I d$ KD significantly affects pathways associated with cell cycle progression $[8,11]$. We first sought to validate this observation in our model system using the pSLIK construct $[12,13]$. We used the metastatic $4 \mathrm{~T} 1$ cell line as it is representative of the TNBC subtype [14], and Id proteins have been shown to play an important role in tumorigenesis in the TNBC subtype. We used an inducible known down system, as reported earlier [1], and observed a significant decrease in the proliferative capacity of cells upon doxycycline (Dox)-induced Id KD in comparison to control conditions.

As proliferation is inextricably linked to the cell cycle, we next characterized the effect of Id KD on cell cycle progression. We found that $I d$ depletion resulted in G0/G1 arrest, as seen in a significant increase in the G0/G1 fraction when compared to the controls (Figure 1a-c). To further elucidate the molecular mechanism through which Id controls the cell cycle, we analyzed the effect of Id KD on the expression of key cell cycle genes that are vital at different phases of the cell cycle. The downregulation of Id significantly decreased the expression of Cyclin A2 (Ccna2), Cyclin B1 (Ccnb1), Cyclin B2 (Ccnb2), Cyclin-dependent kinase1 (Cdk1), and $c-M y c$, as shown in Figure 1d. Interestingly, we found an inverse correlation with $R b$ and $p 21$, which are the negative regulators of these cell cycle genes (Figure 1e).

a.

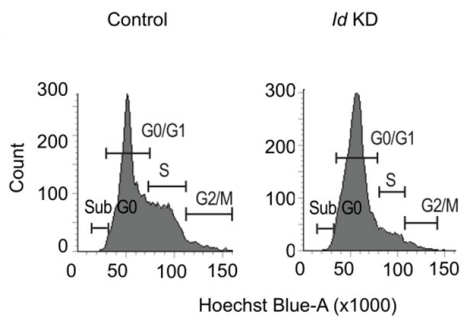

d.

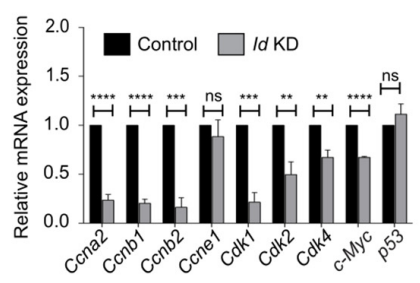

b.

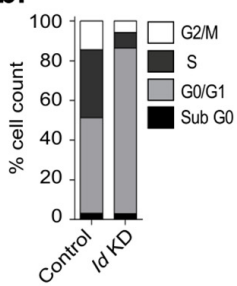

c.

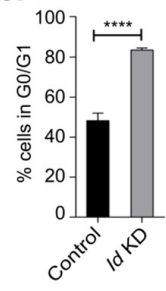

e.

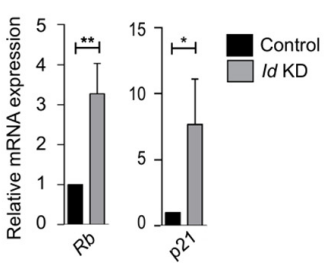

Figure 1. Effect of inhibitor of differentiation (Id) knockdown on cell cycle genes. (a) Flow cytometric analysis of cell cycle on control and Id knockdown (KD) cells after labelling with Hoechst 33342 stain. (b) Comparing the percentage cell count in each phase of the cell cycle, showing a significant increase in the G0/G1 phase in Id KD conditions. (c) Percentage cell count in G0/G1 phase of control and Id KD cells. (d) The relative mRNA expression level of cell cycle genes Cyclin A2, B1, B2, E1 (Ccna2, Ccnb1, Ccnb2, Ccne1), Cyclin dependent kinase 1, 2, 4 (Cdk1,Cdk2,Cdk4), c-Myc, and p53 in control and Id KD cells using qRT-PCR. (e) Relative mRNA expression of Rb and p21 in Id KD cells with respect to control cells were quantified using qRT-PCR. Data were normalized to $\beta$-actinand analyzed by the $2^{-\Delta \Delta C t}$ method. All experiments were performed in three biological replicates and data were expressed as mean \pm standard deviation. Unpaired Student's $t$-test and two-way ANOVA were used. ${ }^{*} p<0.05$, ${ }^{* *} p<0.01,{ }^{* * *} p<0.001,{ }^{* * *} p<0.0001$, ns is non significant. 


\subsection{Identification of Putative Id-Regulated Genes}

To characterize the network of genes regulated by Id proteins, we performed functional annotation analysis on gene array and RNA sequencing data from two different TNBC models of tumor cells marked by either Id depletion or Id1 expression (Figure 2a). The gene array analysis of the Id 1 depletion system has been described previously [1]. The Id1 expression model analyzed genes whose expression was associated with $I d 1$, whereas the $I d$ depletion model attempted to identify downstream targets of Id proteins.

To study the phenotypes associated with depletion of $I d$ as well as to assess its downstream targets, we compared the gene expression profile of three independent replicates of control and Id KD cells by microarray analysis to generate a list of differentially expressed genes between $I d$-depleted and control cells (Table S1). Aiming to discover high confidence genes involved in the Id gene regulatory network, we compared lists of differentially expressed genes in the Id-depleted TNBC model and their controls using MetaCore ${ }^{\mathrm{TM}}$ software. The data were uploaded in MetaCore and filtered using an adjusted $p$-value threshold of 0.05 , resulting in 4301 network objects that were differentially expressed between the $I d \mathrm{KD}$ and the control cells (Figure 2a). To characterize the network of genes regulated by $I d$, we performed enrichment analysis on the candidate genes, which was visualized by process networks and pathway maps. In addition, we used the Id1C3Tag model system to prospectively isolate Id1+ cells, as described previously [1]. The gene expression profiles of the Id1+ and Id1- cells from three independent Id1C3Tag tumors were compared by RNA sequencing exclusively in this study. This resulted in a list of differentially expressed genes between the Id+ and Id- mouse TNBC cells (Table S2). Similarly, the Id1C3Tag RNA sequencing data revealed 126 network objects differentially expressed between Id1+ and Id1- cells (Figure 2a). Interestingly, when we looked at the pathway analysis generated from the differentially expressed gene lists of both models, cell cycle pathways were among the top hits (Figure S1a-c).

By comparing these two datasets, we generated lists of MetaCore network objects common to both experiments as well as those unique to each of the two datasets (Figure 2b). When comparing these lists of network objects from the two TNBC models, we identified 34 high confidence MetaCore network objects as common to both the datasets of differentially expressed genes. Finally, the genes were mapped to network objects in MetaCore, resulting in a list of 26 genes that were significantly regulated in both models of TNBC (Table 1).

We first analyzed the pathways controlled by the 26 putative $I d$ targets. Interestingly, the main pathways regulated by $I d$ involved the cell cycle, specifically the metaphase checkpoint, spindle assembly, and chromosome segregation (Figure 2b,c). Disruption of checkpoint control and aberrant regulation of the cell cycle are observed in tumorigenesis [7,15], resulting in uncontrolled cell proliferation. A key function of $I d$ is the stimulation of cell cycle progression and proliferation by controlling the activity of cell cycle regulators. Studies have already reported that overexpression of $I d$ has been associated with upregulated cell cycle progression in tumorigenesis [7,8,11]. Pathways involving cytoskeleton remodeling, integrin-mediated cell adhesion, migration, and chemotaxis, which are all key steps in epithelial to mesenchymal transition (EMT) and metastasis, were also enriched. Analysis of each individual experiment, along with the genes common to both datasets, showed a similar result, with Id depletion mainly affecting the cell cycle pathway, DNA damage, checkpoints, and cytoskeleton remodeling. Id1 expression model alone showed enrichment for pathways involving hypoxia-induced epithelial-mesenchymal transition, WNT pathway in development, cytoskeleton remodeling, and cell cycle (Figure 2c).

We also looked at the Epithelial-Mesenchymal Transition (EMT) program, which is an important driver of the CSC state, and interestingly found a change in both the E-cadherin and vimentin protein levels (Figure S2a,b). However, when we analyzed the EMT scores of these samples using an inferential scoring metric [16], it did not show any significant change, indicating that the EMT status of the cells was not altered upon Id KD (Figure S2c). 
a.

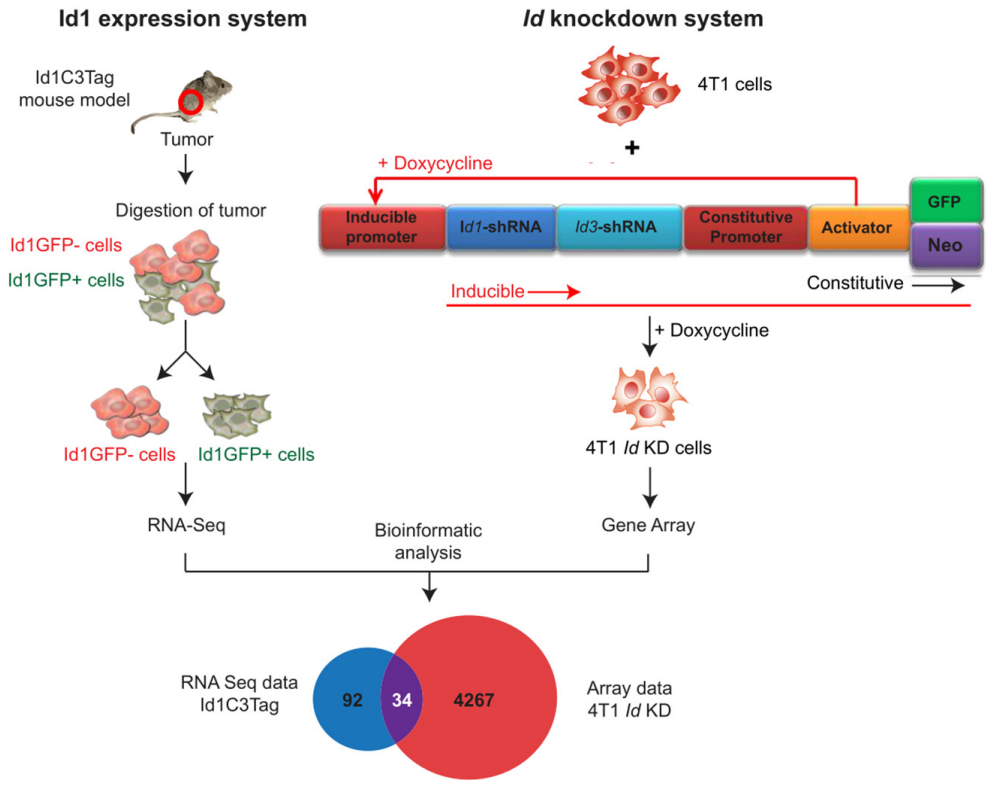

b.

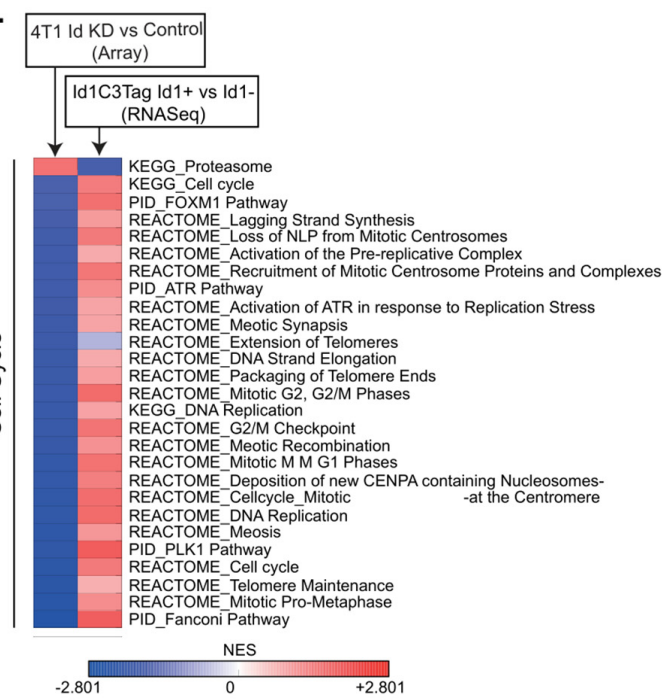

c.

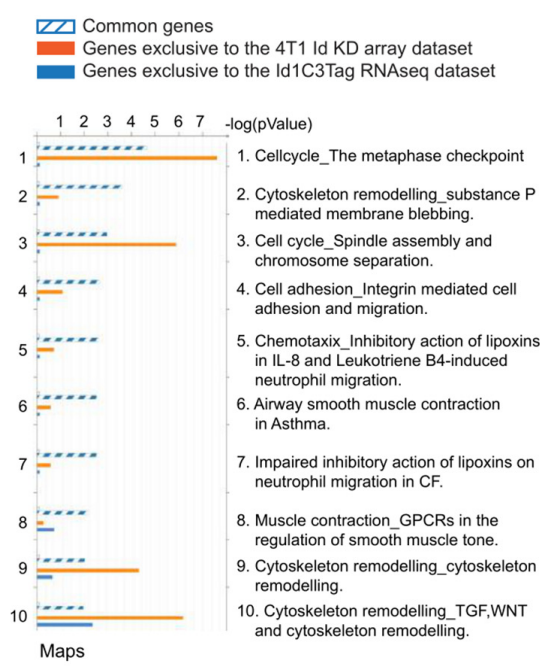

Figure 2. Identification of putative Id-regulated genes. (a) To characterize the network of genes regulated by Id proteins, we performed functional annotation analysis on gene array and RNA sequencing data from two different triple-negative breast cancer (TNBC) models marked by either Id depletion or Id1 expression. Aiming to discover high confidence genes involved in the Id gene regulatory network, we compared lists of differentially expressed genes between the Id-expressing or -depleted TNBC models and their controls using MetaCore software. By comparing these two datasets, we generated lists of MetaCore network objects common to both experiments, as well as those unique to each of the two datasets. The data were uploaded in MetaCore and filtered using an adjusted $p$-value threshold of 0.05 , resulting in 4301 network objects that were differentially expressed between the Id KD and the 4T1 control cells. Similarly, the Id1C3Tag RNA sequencing data revealed 126 network objects specific to mouse differentially expressed between Id+ and Id- cells. Finally, the genes were mapped to network objects in MetaCore, resulting in a list of 34 genes that were significantly differentially regulated in both models of TNBC. (b) To characterize the network of genes regulated by Id, we performed enrichment analysis on the 34 candidate genes, which was visualized by process networks and pathway maps. The enriched pathways included cell cycle, cytoskeleton remodeling, integrin-mediated cell adhesion/migration, and chemotaxis, which are all key steps in epithelial to mesenchymal transition (EMT) and metastasis. (c) The cell cycle pathway had the highest score in the genes common to both datasets analyzed, which correlated with the G0/G1 block observed in Id KD cells. 
Table 1. List of differentially expressed genes common to Id1 expression and Id-depleted model system. The genes highlighted in green were used in further analysis as putative $I d$ targets.

\begin{tabular}{|c|c|c|c|c|c|}
\hline \multirow[b]{2}{*}{$\#$} & \multirow[b]{2}{*}{ Input IDs } & \multicolumn{2}{|c|}{$I d \_K D \_4 T 1 \_m e t a c o r e$} & \multicolumn{2}{|c|}{ Id1C3Tag_RNASeq_metacore } \\
\hline & & Signal & $p$-Value & Signal & $p$-Value \\
\hline 1 & Adamtsl3 & -0.3927 & 0.020463 & 1.3402333 & 0.03359618 \\
\hline 2 & Casc5 & -1.0215 & 0.001402 & 0.9961643 & 0.002269021 \\
\hline 3 & Aspm & -1.3871 & 0.000268 & 0.9586754 & 0.000409133 \\
\hline 4 & Aurka & -1.1447 & 0.0005806 & 0.9813335 & 0.01574964 \\
\hline 5 & Casz1 & 0.2437 & 0.0479447 & 0.9948092 & 0.002230733 \\
\hline 6 & Cenpf & -0.9544 & 0.001877 & 0.7718833 & 0.02742762 \\
\hline 7 & Ctla2a & 0.4903 & 0.0023071 & 1.3066035 & 0.04998167 \\
\hline 8 & Cxcl15 & 2.0181 & 0.0004083 & -6.916629 & 0.01322961 \\
\hline 9 & Angptl7 & -0.631 & 0.0023779 & -1.489744 & 0.002084568 \\
\hline 10 & Cldn6 & -0.5278 & 0.0131683 & -7.315199 & 0.004137659 \\
\hline 11 & Gpr133 & 0.284 & 0.0448178 & -4.065336 & 0.000459154 \\
\hline 12 & Hmga1;Hmga1-rs1 & -0.4779 & 0.0332492 & -1.080467 & 0.001750527 \\
\hline 13 & Il6 & 1.2691 & 0.0003497 & 1.2769696 & 0.004137659 \\
\hline 14 & Kif4 & -1.0266 & 0.0007331 & 0.7764488 & 0.01698252 \\
\hline 15 & Kif11 & -1.3438 & 0.0002452 & 0.7487605 & 0.02222188 \\
\hline 16 & Lphn1 & 0.4649 & 0.0027409 & 0.8679558 & 0.04907909 \\
\hline 17 & $L t b p 2 ; L t b p 3$ & 0.2667 & 0.0244267 & 0.9104315 & 0.002134988 \\
\hline 18 & Mylk & 0.5396 & 0.0154085 & 1.1309435 & 0.00340821 \\
\hline 19 & Lnp;Nusap1 & -1.011 & 0.0001961 & 1.0915483 & 0.008424267 \\
\hline 20 & $P d g f c$ & 1.7513 & 0.00001065 & 0.7934828 & 0.03592082 \\
\hline 21 & Angptl2;Angptl4 & 3.5985 & $5.221 \times 10^{-7}$ & -1.456264 & $1.46715 \times 10^{-5}$ \\
\hline 22 & Prc1 & -0.9945 & 0.0009206 & 0.7781182 & 0.02082709 \\
\hline 23 & Stc2 & -1.2088 & 0.0005806 & -2.135721 & $8.46387 \times 10^{-5}$ \\
\hline 24 & Mylk & 0.5396 & 0.0154085 & 1.1309435 & 0.00340821 \\
\hline 25 & Ube $2 c$ & -0.6684 & 0.0060464 & 1.2384745 & 0.004860396 \\
\hline 26 & Upp1 & -0.5451 & 0.0022003 & -1.767675 & 0.000253826 \\
\hline
\end{tabular}

\subsection{Identification of Kif11 and Aurka as Potential Id Targets}

Among the 26 differentially expressed genes common to the two TNBC models, we prioritized 16 genes for validation. These were chosen on the basis of a significant $p$-value $(<0.05)$ and at least 1.5-fold up- or downregulation compared to the controls. Most of these genes had opposite regulation in the two TNBC models, which was consistent with the fact that one model was marked by Id depletion whereas the other was an Id1 expression model. In addition, eight potential cancer stem cell markers and genes previously implicated in Id biology were chosen on the basis of cell surface localization, significant enrichment in Id+ cells, and availability of antibodies. Altogether 61 candidate genes were identified for further validation as putative $I d$ candidate target genes (Table S3).

We next went on to assess the role of the candidate targets of Id proteins on the proliferative phenotype using a targeted siRNA screen. Interestingly, the target genes that showed the greatest effect on the viability and thus the proliferative phenotype of the Id KD cells with more than $50 \%$ were Kif11, Casc5, Ccnd1, and Aurka (Figure 3a, Table S3). The hits at the other end of the scale included Robo1, which has been investigated in our work separately [1]. 
a.

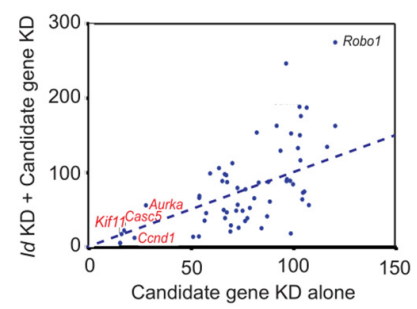

b.

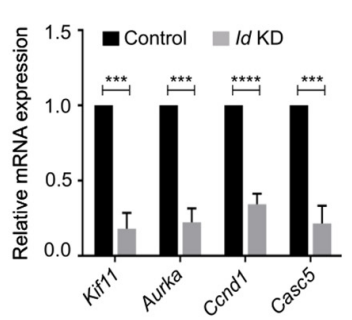

c.

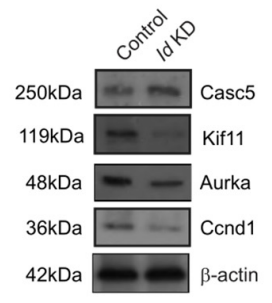

d.

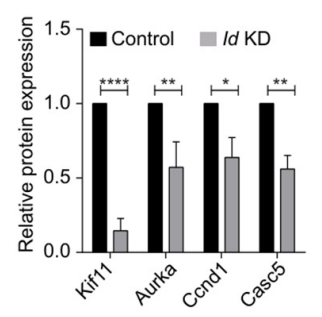

e.

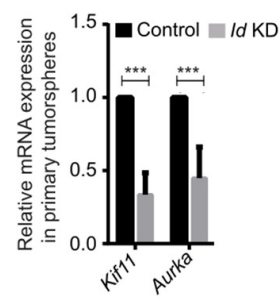

f.

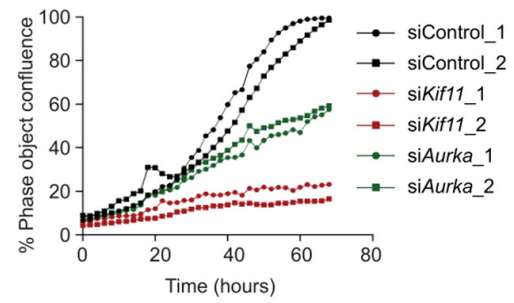

Figure 3. Effect of Id target gene knockdown on the proliferative phenotype. (a) The effect of the candidate genes on proliferation of $4 \mathrm{~T} 1$ cells were assessed by reverse transfection with a $40 \mathrm{nM}$ siGENOMESMART pool siRNA against each of the 61 genes. The target genes that showed the greatest effect on the viability and thus the proliferative phenotype of the $4 \mathrm{~T} 1$ cells with more than $50 \%$ were Kif11, Aurka, Ccnd1, and Casc5. (b) The relative mRNA expression level of Kif11, Aurka, Ccnd1, and Casc5 in Id KD cells with respect to control cells were quantified with qRT-PCR. Data were normalized to $\beta$-actin and analyzed by the $2^{-\Delta \Delta C t}$ method. (c) The expression of the Id target genes Kif11, Aurka, Cond1, and Casc5 at the protein level was decreased in Id KD cells when compared to the controls. (d) Quantification of relative protein expression of Kif11, Aurka, Ccnd1, and Casc5 in control and Id KD normalized with $\beta$-actin. (e) Relative mRNA expression level of Kif11 and Aurka in primary tumourspheres. (f) Percentage confluency in Kif11 KD and Aurka KD conditions, showing significant decrease in cell proliferation compared to the controls using two independent pMission siRNAs per gene. All experiments were performed in three biological replicates and data were expressed as mean \pm standard deviation. Unpaired Student's $t$-test and two-way ANOVA were used. ${ }^{*} p<0.05,{ }^{* *} p<0.01$, $* * * p<0.001, * * * * 0<0.0001$.

To confirm putative Id targets such as Kif11, Aurka, Ccnd1, and Casc5, we analyzed relative mRNA levels. We observed significant reduction in the mRNA levels of Kif11, Aurka, Cyclin D1 (Ccnd1), and Cancer susceptibility candidate 5 (Casc5) in Id KD compared to the controls (Figure 3b). We also detected a decrease in the expression of Kif11, Aurka, Ccnd1, and Casc5 at the protein level by Western blot (Figure 3c,d). Kif11 and Aurka were also downregulated the transcript level in tumorspheres generated in the Id KD cells when compared to control (Figure 3e, Figure S2d).

To confirm the effect of putative $I d$ targets on proliferation, we used an independent pMission siRNA system in 4T1 parental cells. Loss of Kif11 and Aurka lead to significant decrease in the proliferative capacity of the 4T1 cells (Figure 3f, Figure S3a). We continued our studies with Kif11 and Aurka as we did not observe any significant loss of proliferative phenotype with Casc5 and Ccnd1 knockdown (Figure S3b), although we did observe a decrease at the mRNA level (Figure S3c).

\subsection{Kif11 or Aurka Depletion Did Not Phenocopy Loss of Id}

We next characterized the effect of Kif11 KD and Aurka KD on the cell cycle using the pMission system. We found that Kif11 and Aurka depletion lead to cell cycle arrest in the G2/M phase, as evidenced by cell cycle analysis (Figure $4 \mathrm{a}-\mathrm{c}$ ). Intriguingly, this observation was fundamentally different from Id KD case, where it undergoes G0/G1 arrest (Figure 1a, Figure S3e). 
a.

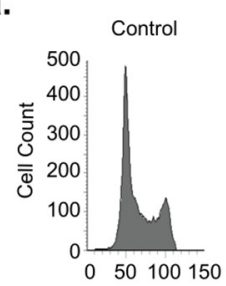

d.
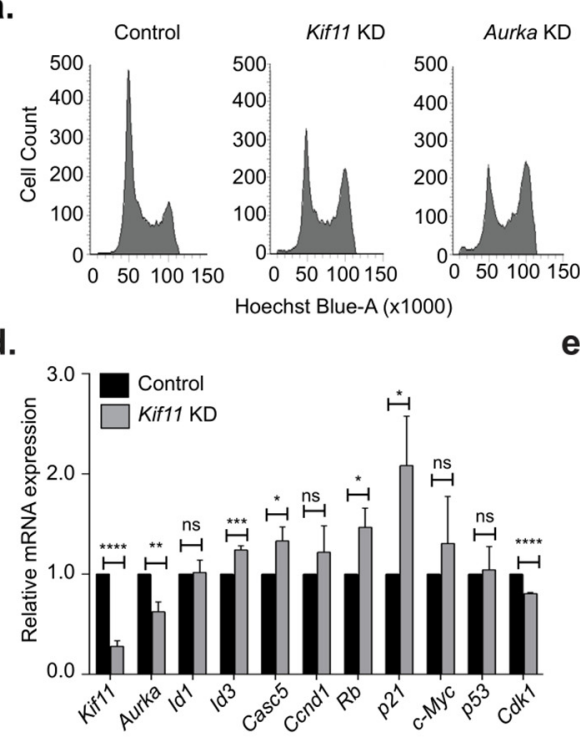

f.
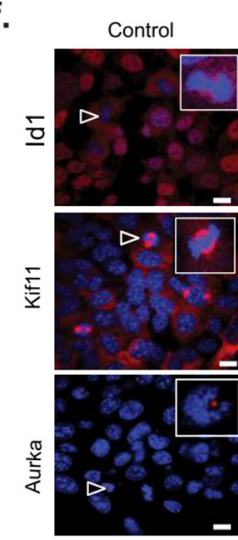

e.

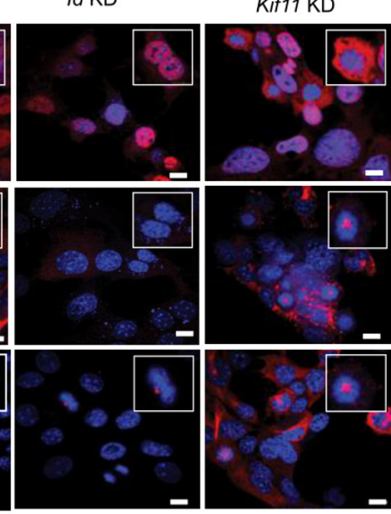

b.

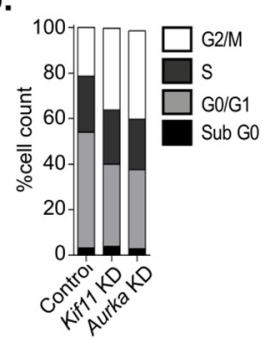

c.

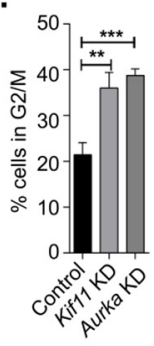

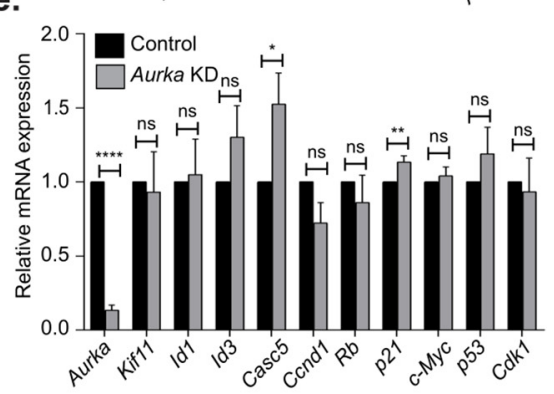

Aurka KD

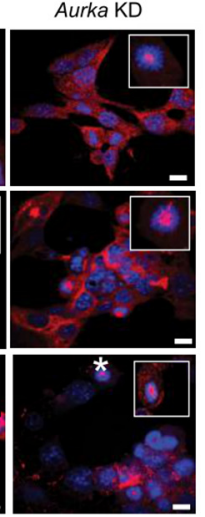

g.

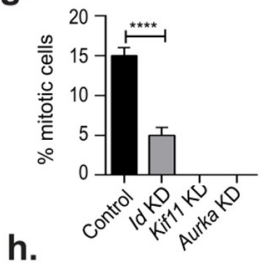

h.

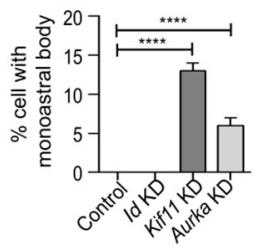

Figure 4. Kif11 and Aurka depletion did not phenocopy loss of Id1. (a) Flow cytometric analysis of cell cycle in Kif11 KD and Aurka KD with scrambled control in 4T1 cells. (b) Comparing the percentage cell count in each phase of the cell cycle after knocking down Kif11 and Aurka. (c) There were significantly higher cells in G2/M phase Kif11 KD and Aurka KD when compared to control. (d,e) The relative mRNA expression level of important cell cycle gene were analyzed in Kif11 KD and Aurka KD with respect to scrambled control using qRT-PCR. Data were normalized to $\beta$-actin and analyzed by the $2^{-\Delta \Delta \mathrm{Ct}}$ method. (f) Immunofluorescence staining for Id1, Kif11, and Aurka on 4T1 control, Id KD, Kif11 KD, and Aurka KD cells. Representative images were taken using a Nicon A1R+ confocal system at 100x magnification with a scale bar corresponding to 100um. 4',6-Diamidino-2-fenylindool (DAPI) shows the nuclear staining; asterisk $\left({ }^{*}\right)$ shows the monoastral bodies formation in siRNA KD system; $\Delta$ shows normal cell division; and inset shows the $100 \times$ zoomed images of the same images. (g) Percentage mitotic cells in control, Id KD, Kif11 KD, and Aurka KD cell lines. (h) Cells exhibiting monoastral body formation in control, Id KD, Kif11 KD, and Aurka KD conditions. All experiments were performed in three biological replicates and data were expressed as mean \pm standard deviation. Unpaired Student's $t$-test and two- way ANOVA were used. ${ }^{*} p<0.05,{ }^{* *} p<0.01,{ }^{* * *} p<0.001,{ }^{* * * *} p<0.0001$, ns is non significant.

To determine the molecular effect of Kif11 and Aurka on cell cycle, we analyzed relative mRNA levels of key cell cycle genes. Kif11 KD significantly reduced the expression of Aurka, even though the expression of Id1 was not altered (Figure 4d). Kif11 depletion had a positive effect on the mRNA levels of $I d 3, C a s c 5, R b$, and $p 21$ and reduced the expression of $C d k 1$, but had no significant effect on Ccnd1, $c-M y c$, and p53. Aurka KD did not show any effect on Id1, Id3, Kif11, or Ccnd1, while there was 
an increased expression of Casc5 and p21 (Figure 4e). We next compared our microscopic observations on the phenotype of the Id KD system with that of Kif11 KD and Aurka KD. We noticed monoastral bodies with improperly assembled mitotic spindle, indicating that the majority of the Kif11 KD and Aurka KD cells were arrested in the M phase of the cell cycle (Figure 4f, Figure S3d). The formation of monoastral bodies is indicative of duplicated chromatin $(4 \mathrm{~N})$ not being able to separate due to perturbations in the spindle formation and centrosome, thus indicating G2/M arrest and matching the cell cycle analysis [17] (Figure 4a). Quantification of percentage mitotic cells (Figure 4g) and cells exhibiting monoastral body phenotype (Figure 4h) in Kif11 KD and Aurka KD clearly demonstrated that Id depletion resulted in a phenotype that is distinct from Kif11 and Aurka.

\subsection{Therapeutic Targeting of CSCs Through Id-Kif11 Axis}

There is currently no effective targeted therapy for TNBC, and chemotherapy is usually the first line of treatment, with a relapse rate of 25\% [18]. Our previous work has demonstrated that Id is critical for CSC-associated phenotypes in TNBC such as proliferation, self-renewal, migration, and metastasis [1]. We have now identified that these phenotypes are influenced by the Id-Kif11/Aurka axis. We hypothesized that targeting Kif11 or Aurka to block the Id1-Kif11/Aurka axis may cause the $I d$-expressing CSC to be more vulnerable to chemotherapy and more effectively debulk the entire tumor mass.

To test this hypothesis, we first determined the $\mathrm{IC}_{50}$ values for two commonly used chemotherapy drugs in breast cancer treatment, paclitaxel and doxorubicin, in 4T1 cells (Figure S4a). Interestingly, we found that there was a significant enrichment for Id1+ tumor cells after treatment with paclitaxel and doxorubicin, suggesting that the Id1+ CSCs are chemoresistant (Figure 5a,b). We next determined the $\mathrm{IC}_{50}$ values for the small molecule inhibitors of Kif11 and Aurka, ispinesib, and alisertib (Figure S4a). Cells treated with ispinesib showed a significant reduction in the percentage of Id1+ cells but no significant change was observed in those treated with alisertib as compared to the control (Figure 5c). We decided to continue with paclitaxel and the Kif11 inhibitor, ispinesib, on the basis of these results.

We next asked whether ispinesib can increase the sensitivity of Id1+ cells to paclitaxel. We found that the cells treated with a combination of paclitaxel and ispinesib showed a significant decrease in cell viability as well as Id1 and Kif11 expression when compared to paclitaxel or ispinesib alone (Figure 5d, Figure S4b-d). Interestingly, we found that the protein level expression of Kif11 was also significantly reduced when treated with the combination (Figure $S 4 b, d$ ).

We next checked the effect of combination therapy on the self-renewal phenotype. A significant reduction in the self-renewal capacity was observed in cells treated with the combination of paclitaxel and ispinesib when compared to either of the drugs alone (Figure 5e,f). To conclude, we have demonstrated that treatment with conventional chemotherapeutic drugs such as paclitaxel enriched Id+ cancer stem cells, which could result in tumor relapses in patients in the clinic. Inhibition of the Id target Kif11 (with ispinesib treatment) in combination with chemotherapy also resulted in a loss of Id+ CSC subpopulations of tumor cells, ultimately leading to more effective debulking of the entire tumor (Figure 5g).

The correlation between ID1 and KIF11 gene expression and relapse free-survival of breast cancer patients was analyzed by the KM Plotter database. TNBC patients with a higher mean gene expression of ID1 and KIF11 together had poor prognosis (HR-1.91, p-value $<0.0082$ ) when compared to patients with low expression of both genes (Figure S4e). This work opens up the possibility of using the KIF11 inhibitor to improve prognosis in patients having with expression of both ID1 and KIF11 using a combination of chemotherapy and small molecule inhibitors such as ispinesib. 


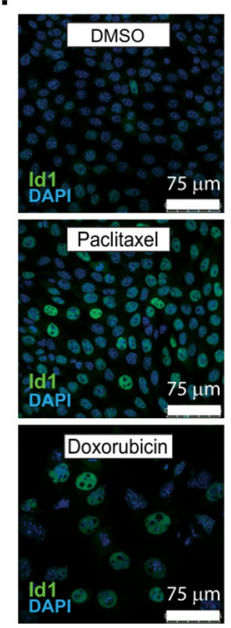

e.

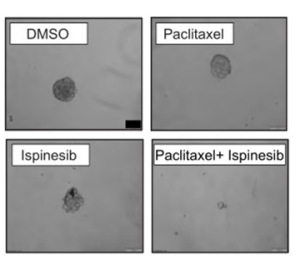

b.

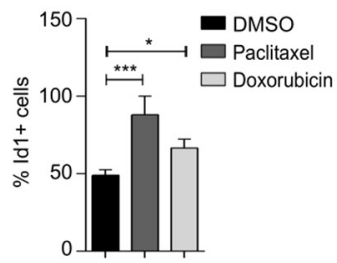

c.

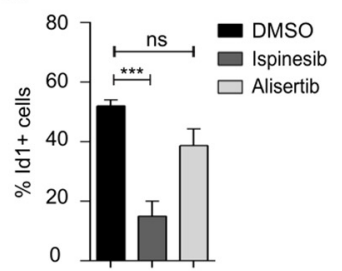

d.

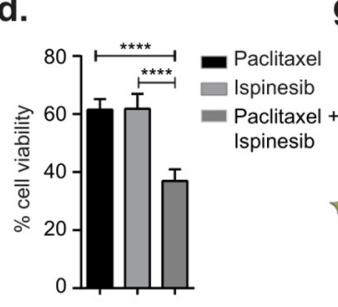

g. Conventional Chemotherapy
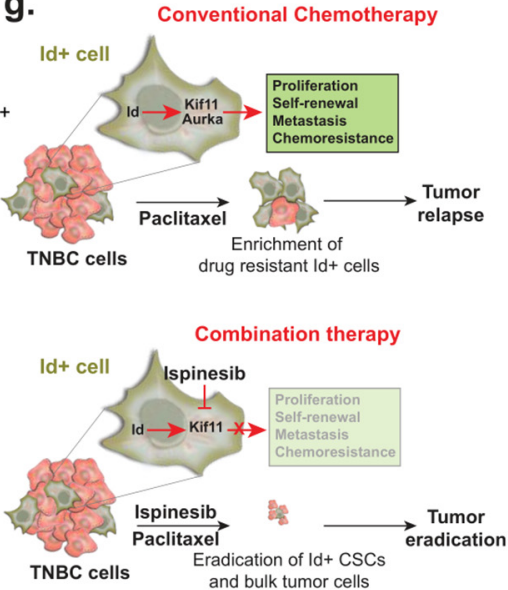

Figure 5. Therapeutic targeting of cancer stem cells (CSCs) through the Id1-Kif11/Aurka axis. (a) Immunofluorescence images of cells to determine Id1 expression after treatment with paclitaxel and doxorubicin (20x). (b) Percentage cells expressing Id1 in control, paclitaxel-, and doxorubicin-treated cells as well as ispinesib and alisertib (c) were determined by immunofluorescence. (d) Cell viability was determined after treating the cells with paclitaxel, ispinesib, and the combination therapy of paclitaxel + ispinesib. Representative phase contrast images of tumor spheres (e) and quantification of number of tumor spheres per 1000 cells (f) in control, paclitaxel, ispinesib, and paclitaxel + ispinesib. (g) Schematic showing the targeting of Id-Kif11 axis with conventional and combination therapy leading to more effective eradication of the entire tumor. All experiments were performed in three biological replicates and data were expressed as mean \pm standard deviation. Unpaired Student's $t$-test and two-way ANOVA were used. ${ }^{*} p<0.05,{ }^{* * *} p<0.001,{ }^{* * * *} p<0.0001$, ns is non significant.

\section{Discussion}

The current body of work sheds light on the role that Id proteins (specifically Id1 and Id3) play in affecting key CSC phenotypes such as proliferation and self-renewal through multiple mechanisms. We observed a striking G0/G1 cell cycle arrest when cancer cells were depleted of Id proteins. By regulating the expression of critical cell cycle genes, Id pauses or checks the cells in the G1 state in a manner that they can re-enter the normal cell cycle once the stress is removed [1]. This supports the theory of $I d$ as a master regulator that, on sensing unfavorable conditions, can "brake" the cells in the G1 phase through multiple means (molecular regulation of cell cycle genes, DNA division inhibition, protein complex perturbation at the centrosome, and spindle fibers). This strategy would allow cells to survive in a state of stasis until conditions favorable to growth of the tumor cell set in.

The idea that CSCs are more plastic and can exist in more than one state may be supported by looking at the Epithelial-Mesenchymal Transition (EMT) program [18]. From the point of view of the EMT scores, we observed that the $I d \mathrm{KD}$ is not pushing the cells clearly towards a more $\mathrm{E}$ or a more $\mathrm{M}$ state, as the levels of both canonical markers decreased. Moreover, our bioinformatic model used a ratio of Ecadherin (CDH1)/Vimentin (VIM) as a predictor to calculate the scores; thus, the relatively proportional change that we saw at RNA/protein levels for both CDH1 and VIM was consistent. The data adds to the evidence that EMT and Mesenchymal-Epithelial Transition 
(MET) are not binary [19] for different stages of EMT and their varying degrees of causal contribution to metastasis.

Using two independent models of $I d 1$ gene expression and gene depletion, we were intrigued to identify the critical cell cycle genes, Kif11 and Aurka, as Id putative gene targets. Previous work in nasopharyngeal cells has linked Id1 and Aurka mechanistically in the induction of tetraploidy. Id1 was found to affect Aurka degradation, which normally occurs during exit from mitosis by the APC/C Cdh1-mediated proteolysis pathway. Id1 stabilized Aurka by active competition with Cdh1, thus preventing Aurka degradation [20]. Interestingly, while individual knockdown of Kif11 and Aurka also led to a proliferative arrest, it did not phenocopy the G0/G1 cell cycle arrest with the Id knockdown or the formation of monoastral bodies. This suggested that the impediment of the cell cycle by Id protein is through different mechanisms and not the canonical mitotic pathways involving the microtubules by Kif11/ Aurka, which forms a part of our future investigation.

There is no targeted therapy for TNBC, and chemotherapy is the first line of treatment. Thus, we checked the effect of the commonly used chemotherapy drugs paclitaxel and doxorubicin, which are used in the clinic for treatment of TNBC. Studies by our group and others have already reported that Id1 marks a chemoresistant breast cancer cell [7] in cancers such as hepatocarcinomas [21], and the Kif11 pathway has been targeted in the treatment of docetaxel-resistant TNBC cells [22]. However, the most compelling reason to target the Id1/Kif11 pathway came from work [23] that identified the drug BRD9876 as a kinesin-5 inhibitor in multiple myeloma, which led to significant downregulation of ID1. On the basis of our work, we used Id as a marker for the chemoresistant CSC population in TNBC. We tested our hypothesis that we can achieve a better response by combining traditional chemotherapy along with ablation of the Id-expressing chemoresistant cells using small molecule inhibitors against the Id target Kif11. We achieved a significant decrease in proliferative and self-renewal capacity when the cells were treated with paclitaxel and ispinesib by successfully targeting subpopulations of cells, including the Id+CSCs within a tumor.

A key limitation of our study is that we focused on two genes on the basis of the phenotype affected. Future work involves dissecting the role of other putative Id targets along with combinatorial therapy using a wider range of commonly used chemotherapeutic drugs on the basis of the Id targets validated.

Thus, a combination of targeted drugs with chemotherapy would be an effective strategy for the complete treatment of TNBC and would give women currently living with this disease a better long-term prognosis.

\section{Conclusions}

CSCs are drivers of tumor progression and metastasis in breast cancer. Using two model systems with a combined transcriptomic analysis and large scare siRNA screen, we demonstrated the important role of the Id proteins in the cell cycle process, which is corrupted in a cancer context by Id targets such as Kif11 and Aurka. This has importantly led to the identification of the Kif11 small molecule inhibitor ispinesib to eradicate the Id1+ cells that are resistant to paclitaxel. Demonstrating the effectiveness of combination chemotherapy with targeted drug therapy against the Id1+ CSC opens up new avenues to explore. Future work involves dissecting the role of other putative Id targets along with combinatorial testing with chemotherapeutic drugs to more effectively debulk the entire tumor cell population.

\section{Data Availability}

The datasets generated for this study can be found in the GEO Database GSE129790, GSE129858, and GSE129859.

Supplementary Materials: The following are available online at http://www.mdpi.com/2218-273X/10/9/1295/s1, Figure S1: Identification of putative Id regulated genes, Figure S2: Effect of Id knockdown on EMT markers and selfrenewal, Figure S3: Kif11 and Aurka are Id targets, Figure S4: Determining the IC50 values for chemotherapy and small molecule inhibitors, Table S1: List of top 50 DE genes between control and Id depleted cells, Table S2: List of differentially expressed genes between the Id+ and Id- mouse TNBC cells generated from the Id1C3Tag model. 
Author Contributions: R.N. contributed to the conceptualization and execution of the project. A.P.T., R.M., B.A.V., and N.K. contributed to the methodology. D.L.R. was involved in the genomic analysis. R.M., A.P.T., B.A.V., N.K., W.S.T., A.C., E.D., A.M., C.V.K., H.H., J.Y., and L.A.B. contributed to the investigations. R.N. wrote the original draft of the manuscript. J.T.G., H.L., and M.K.J. contributed to the bioinformatic analysis of the EMT program. A.S. reviewed and edited the manuscript. A.S. and R.N. contributed to the funding acquisition. All authors have read and agreed to the published version of the manuscript.

Funding: This work was supported by funding from the Early Career Research (ECR) Award from Science and Engineering Research Board (SERB), Government of India (ECR/2015/000031), and in part from the National Health and Medical Research Council (NHMRC) of Australia. R.N. is the recipient of the Ramanujan Fellowship from the Government of India (SERB) (SB/S2/RJN/182/2014). A.S. is the recipient of a Senior Research Fellowship from the NHMRC. S.O.T. is supported by National Breast Cancer Foundation (NBCF) practitioner fellowship. A.P.T. and B.A.V. are funded through the Council of Scientific and Industrial Research (CSIR) Junior Research Fellowship and Department of Science and Technology (DST) INSPIRE Fellowship, respectively.

Acknowledgments: We would like to thank the following people for their assistance with this manuscript: Tilak Prasad and Surabhi S.V. for helping with flow cytometry, Anurup K.G. for technical support with confocal imaging, and T. R. Santhosh Kumar for helping with lab consumables and technical support.

Conflicts of Interest: The authors declare no competing interests.

\section{Abbreviations}

CSC cancer stem cell

TNBC triple negative breast cancer

Id inhibitor of differentiation

\section{References}

1. Teo, W.S.; Holliday, H.; Karthikeyan, N.; Cazet, A.S.; Roden, D.L.; Harvey, K.; Konrad, C.V.; Murali, R.; Varghese, B.A.; Archana, P.T.; et al. Id proteins promote a cancer stem cell phenotype in triple negative breast cancer via negative regulation of Robo1. Front. Cell Dev. Biol. Mol. Cellular Oncol. 2020, 8, 552. [CrossRef]

2. Rivenbark, A.G.; O'Connor, S.M.; Coleman, W.B. Molecular and cellular heterogeneity in breast cancer: Challenges for personalized medicine. Am. J. Pathol. 2013, 183, 1113-1124. [CrossRef]

3. Yam, C.; Mani, S.A.; Moulder, S.L. Targeting the Molecular Subtypes of Triple Negative Breast Cancer: Understanding the Diversity to Progress the Field. Oncology 2017, 22, 1086-1093. [CrossRef]

4. Sharma, P. Biology and Management of Patients with Triple-Negative Breast Cancer. Oncology 2016, 21, 1050-1062. [CrossRef]

5. Phi, L.T.H.; Sari, I.N.; Yang, Y.-G.; Lee, S.-H.; Jun, N.; Kim, K.S.; Lee, Y.K.; Kwon, H. Cancer Stem Cells (CSCs) in Drug Resistance and their Therapeutic Implications in Cancer Treatment. Stem Cells Int. 2018, 2018, 1-16. [CrossRef] [PubMed]

6. Prieto-Vila, M.; Takahashi, R.-U.; Usuba, W.; Kohama, I.; Ochiya, T. Drug Resistance Driven by Cancer Stem Cells and Their Niche. Int. J. Mol. Sci. 2017, 18, 2574. [CrossRef]

7. Nair, R.; Roden, D.L.; Teo, W.S.; McFarland, A.; Junankar, S.; Ye, S.; Nguyen, A.; Yang, J.; Nikolić, I.; Hui, M.; et al. c-Myc and Her2 cooperate to drive a stem-like phenotype with poor prognosis in breast cancer. Oncogene 2013, 33, 3992-4002. [CrossRef]

8. Swarbrick, A.; Åkerfeldt, M.C.; Lee, C.S.L.; Sergio, C.M.; Caldon, C.E.; Hunter, L.-J.K.; Sutherland, R.L.; Musgrove, E.A. Regulation of cyclin expression and cell cycle progression in breast epithelial cells by the helix-loop-helix protein Id1. Oncogene 2004, 24, 381-389. [CrossRef]

9. Livak, K.J.; Schmittgen, T.D. Analysis of relative gene expression data using real-time quantitative PCR and the 2(-Delta Delta C(T)) Method. Methods 2001, 25, 402-408. [CrossRef]

10. Győrffy, B.; Lanczky, A.; Eklund, A.C.; Denkert, C.; Budczies, J.; Li, Q.; Szállási, Z. An online survival analysis tool to rapidly assess the effect of 22,277 genes on breast cancer prognosis using microarray data of 1,809 patients. Breast Cancer Res. Treat. 2009, 123, 725-731. [CrossRef]

11. O’Brien, C.A.; Kreso, A.; Ryan, P.; Hermans, K.G.; Gibson, L.; Wang, Y.; Tsatsanis, A.; Gallinger, S.; Dick, J.E. ID1 and ID3 Regulate the Self-Renewal Capacity of Human Colon Cancer-Initiating Cells through p21. Cancer Cell 2012, 21, 777-792. [CrossRef] [PubMed] 
12. Swarbrick, A.; Roy, E.; Allen, T.D.; Bishop, J.M. Id1 cooperates with oncogenic Ras to induce metastatic mammary carcinoma by subversion of the cellular senescence response. Proc. Natl. Acad. Sci. USA 2008, 105, 5402-5407. [CrossRef]

13. Shin, K.-J.; Wall, E.A.; Zavzavadjian, J.R.; Santat, L.A.; Liu, J.; Hwang, J.-I.; Rebres, R.; Roach, T.; Seaman, W.; Simon, M.I.; et al. A single lentiviral vector platform for microRNA-based conditional RNA interference and coordinated transgene expression. Proc. Natl. Acad. Sci. USA 2006, 103, 13759-13764. [CrossRef]

14. Kaur, P.; Nagaraja, G.M.; Zheng, H.; Gizachew, D.; Galukande, M.; Krishnan, S.; Asea, A. A mouse model for triple-negative breast cancer tumor-initiating cells (TNBC-TICs) exhibits similar aggressive phenotype to the human disease. BMC Cancer 2012, 12, 120. [CrossRef] [PubMed]

15. Caldon, C.E.; Swarbrick, A.; Lee, C.S.; Sutherland, R.L.; Musgrove, E.A. The Helix-Loop-Helix Protein Id1 Requires Cyclin D1 to Promote the Proliferation of Mammary Epithelial Cell Acini. Cancer Res. 2008, 68, 3026-3036. [CrossRef]

16. George, J.T.; Jolly, M.K.; Xu, S.; Somarelli, J.A.; Levine, H. Survival Outcomes in Cancer Patients Predicted by a Partial EMT Gene Expression Scoring Metric. Cancer Res. 2017, 77, 6415-6428. [CrossRef]

17. Hut, H.M.; Lemstra, W.; Blaauw, E.H.; Van Cappellen, G.W.; Kampinga, H.H.; Sibon, O.C. Centrosomes Split in the Presence of Impaired DNA Integrity during Mitosis. Mol. Biol. Cell 2003, 14, 1993-2004. [CrossRef]

18. Luo, M.; Brooks, M.; Wicha, M.S.; Brooke, M. Epithelial-Mesenchymal Plasticity of Breast Cancer Stem Cells: Implications for Metastasis and Therapeutic Resistance. Curr. Pharm. Des. 2015, 21, 1301-1310. [CrossRef]

19. Jolly, M.K.; Ware, K.E.; Gilja, S.; Somarelli, J.A.; Levine, H. EMT and MET: Necessary or permissive for metastasis? Mol. Oncol. 2017, 11, 755-769. [CrossRef]

20. Vaillant, F.; Asselin-Labat, M.-L.; Shackleton, M.; Forrest, N.C.; Lindeman, G.J.; Visvader, J.E. The mammary progenitor marker CD61/beta3 integrin identifies cancer stem cells in mouse models of mammary tumorigenesis. Cancer Res. 2008, 68, 7711-7717. [CrossRef]

21. Liao, X.; Bu, Y.; Jiang, S.; Chang, F.; Jia, F.; Xiao, X.; Song, G.; Zhang, M.; Ning, P.; Jia, Q. CCN2-MAPK-Id-1 loop feedback amplification is involved in maintaining stemness in oxaliplatin-resistant hepatocellular carcinoma. Hepatol. Int. 2019, 13, 440-453. [CrossRef]

22. Jiang, M.; Zhuang, H.; Xia, R.; Gan, L.; Wu, Y.; Ma, J.; Sun, Y.; Zhuang, Z. KIF11 is required for proliferation and self-renewal of docetaxel resistant triple negative breast cancer cells. Oncotarget 2017, 8, 92106-92118. [CrossRef] [PubMed]

23. Chattopadhyay, S.; Stewart, A.L.; Mukherjee, S.; Huang, C.; Hartwell, K.A.; Miller, P.G.; Subramanian, R.; Carmody, L.C.; Yusuf, R.Z.; Sykes, D.B.; et al. Niche-Based Screening in Multiple Myeloma Identifies a Kinesin-5 Inhibitor with Improved Selectivity over Hematopoietic Progenitors. Cell Rep. 2015, 10, 755-770. [CrossRef]

(C) 2020 by the authors. Licensee MDPI, Basel, Switzerland. This article is an open access article distributed under the terms and conditions of the Creative Commons Attribution (CC BY) license (http://creativecommons.org/licenses/by/4.0/). 